\title{
Eyeglass: A Very Large Aperture Diffractive Space Telescope
}

R. Hyde, S. Dixit, A. Weisberg, M. Rushford

This article was submitted to 2002 Astronomical Telescopes \& Instrumentation Conference, Waikoloa, HI., August 22-28, 2002

\section{July 29, 2002}

U.S. Department of Energy

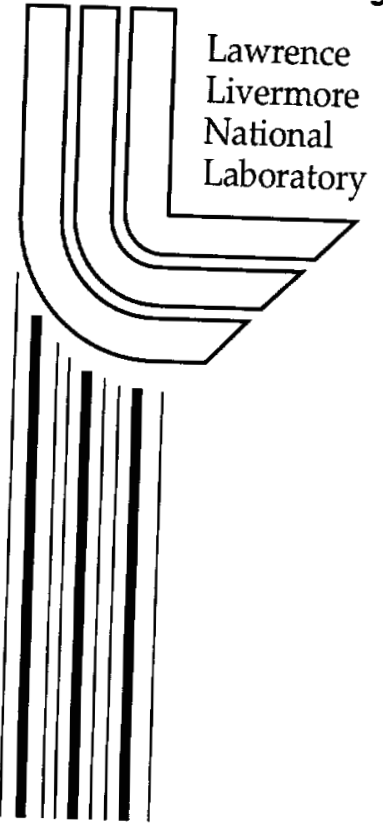




\section{DISCLAIMER}

This document was prepared as an account of work sponsored by an agency of the United States Government. Neither the United States Government nor the University of California nor any of their employees, makes any warranty, express or implied, or assumes any legal liability or responsibility for the accuracy, completeness, or usefulness of any information, apparatus, product, or process disclosed, or represents that its use would not infringe privately owned rights. Reference herein to any specific commercial product, process, or service by trade name, trademark, manufacturer, or otherwise, does not necessarily constitute or imply its endorsement, recommendation, or favoring by the United States Government or the University of California. The views and opinions of authors expressed herein do not necessarily state or reflect those of the United States Government or the University of California, and shall not be used for advertising or product endorsement purposes.

This is a preprint of a paper intended for publication in a journal or proceedings. Since changes may be made before publication, this preprint is made available with the understanding that it will not be cited or reproduced without the permission of the author.

This work was performed under the auspices of the United States Department of Energy by the University of California, Lawrence Livermore National Laboratory under contract No. W-7405-Eng-48.

This report has been reproduced directly from the best available copy.

Available electronically at http://www.doc.gov/bridge

Available for a processing fee to U.S. Department of Energy

And its contractors in paper from

U.S. Department of Energy

Office of Scientific and Technical Information

P.O. Box 62

Oak Ridge, TN 37831-0062

Telephone: (865) 576-8401

Facsimile: (865) 576-5728

E-mail: reports@adonis.osti.gov

Available for the sale to the public from

U.S. Department of Commerce

National Technical Information Service

5285 Port Royal Road

Springfield, VA 22161

Telephone: (800) 553-6847

Facsimile: (703) 605-6900

E-mail: orders@ntis.fedworld.gov

Online ordering: http://www.ntis.gov/ordering.htm

\section{OR}

Lawrence Livermore National Laboratory

Technical Information Department's Digital Library

http://www.llnl.gov/tid/Library.html 


\title{
Eyeglass : A Very Large Aperture Diffractive Space Telescope
}

\author{
Roderick Hyde, Shamasundar Dixit, Andrew Weisberg, Michael Rushford
}

Lawrence Livermore National Laboratory

\begin{abstract}
Eyeglass is a very large aperture ( $25-100$ meter) space telescope consisting of two distinct spacecraft, separated in space by several kilometers. A diffractive lens provides the telescope's large aperture, and a separate, much smaller, space telescope serves as its mobile eyepiece. Use of a transmissive diffractive lens solves two basic problems associated with very large aperture space telescopes; it is inherently fieldable (lightweight and flat, hence packagable and deployable) and virtually eliminates the traditional, very tight, surface shape tolerances faced by reflecting apertures. The potential drawback to use of a diffractive primary (very narrow spectral bandwidth) is eliminated by corrective optics in the telescope's eyepiece. The Eyeglass can provide diffraction-limited imaging with either single-band, multiband, or continuous spectral coverage.

Broadband diffractive telescopes have been built at LLNL and have demonstrated diffractionlimited performance over a $40 \%$ spectral bandwidth $(0.48-0.72 \mu \mathrm{m})$. As one approach to package a large aperture for launch, a foldable lens has been built and demonstrated. A $75 \mathrm{~cm}$ aperture diffractive lens was constructed from 6 panels of $1 \mathrm{~mm}$ thick silica; it achieved diffraction-limited performance both before and after folding. This multiple panel, folding lens, approach is currently being scaled-up at LLNL. We are building a 5 meter aperture foldable lens, involving 72 panels of $700 \mu \mathrm{m}$ thick glass sheets, diffractively patterned to operate as coherent $\mathrm{f} / 50$ lens.
\end{abstract}

\section{INTRODUCTION}

The history of observational astronomy is dominated by the continual quest for larger aperture telescopes. Increased collecting area allows us to gather more light, and increased resolution allows us to more clearly distinguish different objects. Both of these improvements are, by themselves, of major importance: Increased collecting area shortens observation times, thereby allowing the telescope to be used to examine more objects. Increased resolution permits us to view finer features on nearby objects and to sort-out distant targets (for instance, extrasolar planets from their stars, and extragalactic supernova from their hosts). Accordingly there is interest in building future space telescopes which are much larger than Hubble (2.4 m), and its follow-on, NGST $(\sim 6 \mathrm{~m})$; sizes of $25-100$ meters would be very useful.

Unfortunately, fielding large optics in space is exceedingly difficult. The two fundamental difficulties preventing the fielding of large aperture space optics are meeting the tight optical tolerances necessary to achieve high resolution images (furthermore, doing so across large apertures), and simultaneously dealing with the weight, packaging and deployability limitations of space implementation.

At the Lawrence Livermore National Laboratory, we are working to develop a new type of space telescope, based upon the use of diffractive optics, which is designed to simultaneously address both of these fundamental difficulties. 
An Eyeglass space telescope consists of two distinct spacecraft, separated in space by a few kilometers. The telescope's large aperture is provided by a transmissive diffractive lens, acting as a Magnifying Glass. This large lens gathers and gently focuses light, directing it to a distant focal surface, where it is collected by a separate, much smaller, space telescope serving as the Eyeglass's mobile Eyepiece.

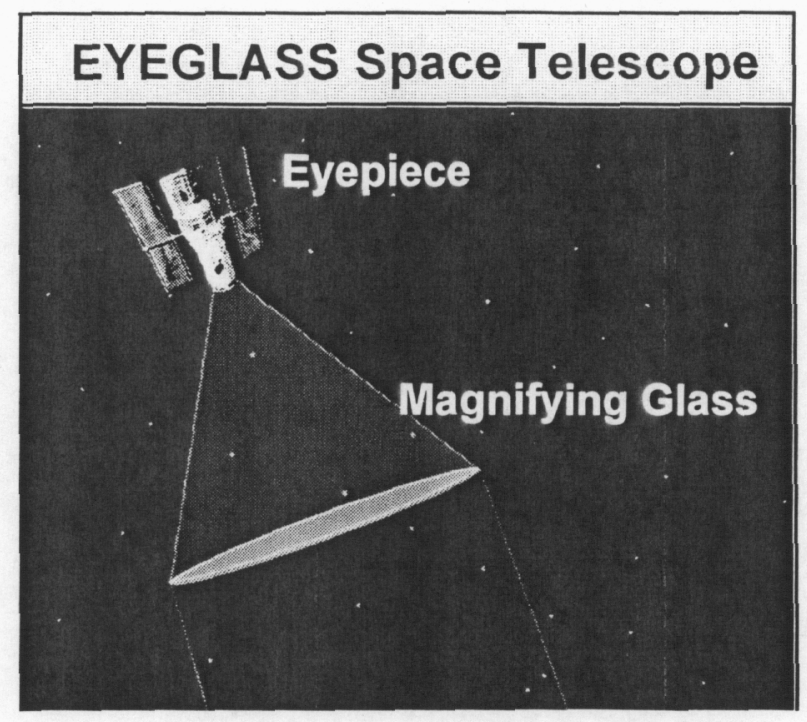

Figure 1: Eyeglass Space Telescope

The use of a transmissive diffractive lens for the Eyeglass's large aperture is the key to solving the optical tolerance and fielding problems generally associated with large space telescopes. Thin transmissive optics are inherently less sensitive to surface shape errors than are reflectors. This increased tolerance to surface ripples comes about because light reflecting off a surface bump picks up a path delay equal to twice the ripple height where as light going through a bump picks up almost no path delay, since the path delay introduced by one surface is nearly completely cancelled by the second surface.
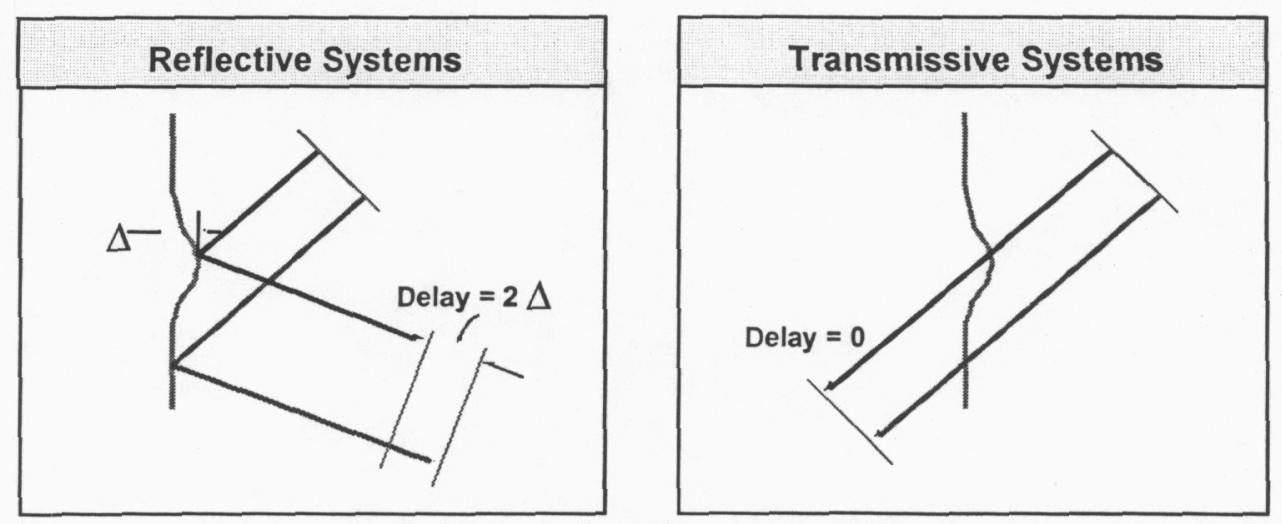

Figure 2: Optical effect of ripples on thin membranes

Quantitatively, a ripple on a reflective membrane designed to focus light through an angle $\theta$ changes the light path by $(1+\cos \theta)$. If the same membrane is used in transmission, then the effect of the ripple is to multiply the light path by $(1-\cos \theta)$. Lenses therefore have a $(1+\cos \theta) /(1-\cos \theta)$ tolerance advantage over mirrors. By making the lens weak, i.e., by keeping 
$\theta$ small, this tolerance gain becomes large, scaling as $4 / \theta^{2}$, i.e., as $16(\mathrm{f} / \#)^{2}$. A slow thin, $\mathrm{f} / 100$ lens can tolerate 160,000 times greater shape errors than a similar mirror. This relaxes surface figure tolerance requirements from hundreds of angstroms up to millimeter levels.

The second problem, the difficulty of fielding objects in-space, is dealt with because of the fact that the Magnifying Glass is a thin flat membrane. The membrane's thinness $(<100 \mu \mathrm{m})$ makes it both lightweight and, because it is flexible, easy to package. Unlike reflective membranes, the lens's focusing power comes from a diffractive surface profile, not a curved shape; this membrane lens operates while flat, and is therefore easy to deploy and hold taut.

The reason for using a diffractive lens as the Eyeglass's large Magnifying Glass is simply that a conventional refractive lens would be thick; it is far too heavy and bulky to be fielded. Diffractive optics (i.e., coherent Fresnel lenses), however, do provide a means to field truly thin transmissive lenses, since they chop the applied phase correction into $2 \pi$-thick sections. This sized phase correction can be performed (in the visible) with a series of $1 \mu \mathrm{m}$ deep quadraticallyblazed surface cuts; even with an underlying substrate, the overall film is thin, packagable, and extraordinarily lightweight; enabling optics with an areal density less than $0.1 \mathrm{~kg} / \mathrm{m}^{2}$.

Unfortunately, an Eyeglass telescope is inherently long; the product of its large aperture and high f/\# (responsible for its tolerance advantages) dictates primary focal lengths of approximately a kilometer. Such lengths are impractical for a single spacecraft, so the Eyeglass telescope is split into two separate vehicles, a Magnifying Glass and an Eyepiece.

The Magnifying Glass vehicle is responsible for supporting the large aperture diffractive lens, holding it in the proper shape and for swiveling it in order to point towards desired targets. Incident light is gathered by the Magnifying Glass's diffractive lens and gently focused towards a distant focal point. There it is collected and imaged by a free-flying Eyepiece vehicle. This compact spacecraft has a modest (meter-scale) aperture; it is quite similar to existing (conventional) space telescopes. This compact spacecraft has a modest (meter-scale) aperture, and is, in most respects, similar to existing space telescopes; but does face two special requirements due to its cooperation with the Magnifying Glass. It is responsible for the stationkeeping necessary to keep the overall Eyeglass telescope in focus and properly pointed, and also performs the chromatic correction necessary to turn this diffractive telescope from a monochromatic to a broad-band instrument.

\section{OPTICAL PERFORMANCE}

Basing a telescope upon a diffractive lens does pose a fundamental optical challenge: Diffractive optics are strongly chromatic, with a focal-length inversely proportional to wavelength. Because of this, they are generally used only for monochromatic (i.e., laser) applications.

In order to make a broad-band diffractive telescope, we must employ some form of chromatic correction. We can not make the Magnifying Glass itself achromatic, so instead will cancel its chromatic aberrations with correcting optics in the separate Eyepiece vehicle. A way of doing this was invented 100 years ago by Schupmann for refractive systems. Fortunately, his technique is general, and applies just as well for diffractive optics. The general layout for a Schupmann-style diffractive telescope is shown below: 


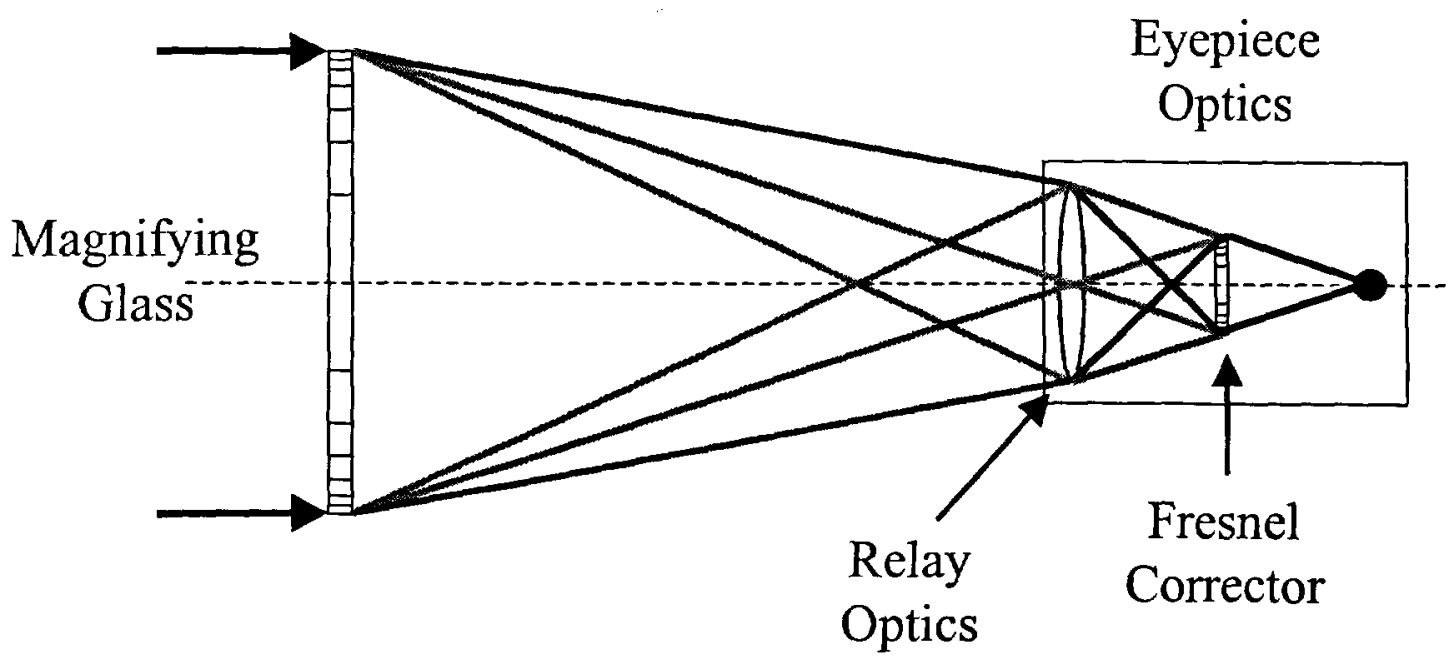

Figure 3: Layout for a color-corrected diffractive telescope

Physically, the reason Schupmann correction works for a diffractive telescope such as Eyeglass is clear; light leaves each point of the Magnifying Glass's diffractive lens in an angular spray, each color being sent into a different direction. As the light from this site travels towards the Eyepiece it spreads apart, diverging both spectrally and physically; both effects must be corrected. The physical reassembly is achieved first, by making the light pass through a relay telescope as it enters the Eyepiece. This internal telescope focuses the surface of the Magnifying Glass onto that of the Fresnel Corrector, thereby physically recombining rays which left each site on the first diffractive lens to a matching site on the second one. Now each site on the Fresnel Corrector sees an incoming angular/color spray corresponding to that from the departure site on the Magnifying Glass; by employing an inverse (defocusing) diffractive profile, it can remove this angular/color spray. As a result, each ray bundle is now both physically and spectrally recombined; the set of bundles can then be brought to a common achromatic focus.

We have built and tested two broad-band diffractive telescopes in order to demonstrate the effectiveness of this color-correction method. Both telescopes utilized a simple transmissive lens (albeit a dual-element achromat) as the relay system.

Our first diffractive telescope was based upon a $20 \mathrm{~cm}, \mathrm{f} / 100$ lens. The lens was fabricated by a lithographic process, generating a 4-level, digitally blazed, phase profile [2]. In order to chromatically correct this diffractive lens, we combined it with a $4 \mathrm{~cm}$ relay lens (a refractive achromat) and a $2.2 \mathrm{~cm}, \mathrm{f} / 11$, inverse diffractive lens.

This experiment successfully demonstrated our ability both to chromatically correct diffractive telescopes, and to do so with diffraction-limited performance. In Figure 4 we show the telescope's point spread function (PSF) for $633 \mathrm{~nm}$ laser light and for broad-band (470-700 nm) white-light. The broadband light is clearly focused into a tight spot, which has the predicted 95 $\mu \mathrm{m}$ Airy width. We also used this telescope (piping in light via turning mirrors) to look at real astronomical objects, taking pictures of the Moon, Saturn, Jupiter, and the Sun.

Next, we used our existing photolithographic tools to make a $50 \mathrm{~cm}$ diameter, $\mathrm{f} / 100,4$-level diffractive lens in a fused silica substrate [2]. We used this silica lens as the primary in a $50 \mathrm{~cm}$ aperture, $\mathrm{f} / 100$, color-corrected, diffractive telescope. The large diameter and slow $\mathrm{f} /$ number of this lens, led to a $>50 \mathrm{~m}$ long design for the telescope. We used the NOVA laser bay to provide 
the large, vibrationally and environmentally controlled, location needed for fielding this diffractive telescope. The optical design was basically a scaled-up version of the one used for the $20 \mathrm{~cm}$ telescope, and also achieved diffraction-limited broadband focusing.
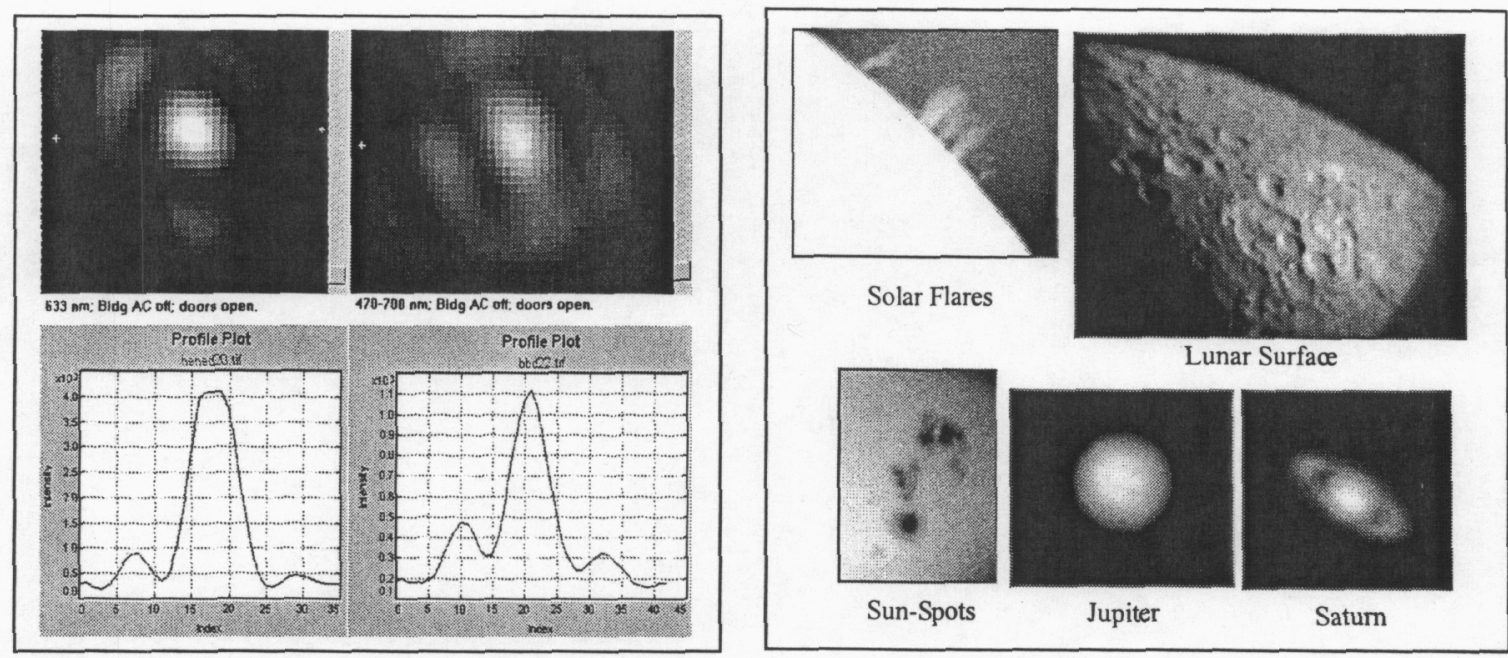

Figure 4: Optical performance for LLNL color-corrected diffractive telescopes

These 20- and 50-cm telescope demonstrations clearly validate the concept of a fully color corrected diffractive telescope. However, achieving similar performance from a large, spacebased telescope is much more difficult.

The first challenge is that the reimaging from Magnifying Glass to Fresnel Correction must be near-perfect; the relay optics themselves be achromatic and aberration-free. This prevents large operational telescopes from using a simple refractive lens relay as shown above. Instead, we have designed sophisticated, off-axis, dual-element, reflective relay systems which will allow diffraction-limited imaging with large Eyeglass telescopes[3].

The second challenge arises from the fact that the Eyepiece collector can only correct the light that it sees. Light begins spectrally spreading apart as soon as it leaves the Magnifying Glass, and that which is sufficiently far off-color will simply miss the Eyepiece. So, an Eyeglass telescope is naturally a single spectral-window device, having a natural bandwidth determined by the aperture ratio between the reimaging system in the Eyepiece and the Magnifying Glass. For large space telescopes, the desire to keep the mass and size of the conventional optics on the Eyepiece reasonably small will force the telescope's natural bandwidth to be much smaller than the $\pm 20 \%$ demonstrated above. The key to attaining wide spectral coverage is via multi-band diffractive telescopes.

Multi-spectral diffractive telescopes are made possible by replacing the standard, mod- $2 \pi$, quadratically-blazed phase-profiles used in the Magnifying Glass and Fresnel Corrector of a single-band Eyeglass with higher-order, $\bmod -2 \pi \mathrm{N}$, profiles. From a fabrication standpoint this is a fairly benign adjustment; the surface features become $\mathrm{N}$-fold deeper and wider, while the tolerance requirements remain unchanged, set by the shortest wavelength to-be-viewed.

Optically, this approach draws upon the fact that a quadratically-blazed diffractive lens

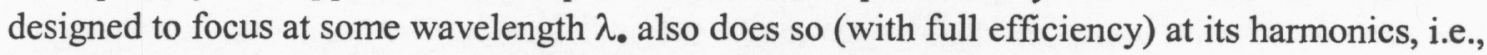
at $\lambda_{\odot} / 2, \lambda_{\odot} / 3, \lambda_{\odot} / 4$, etc. Because the Eyeglass's chromatic correction depends only upon the 
geometrically-fixed, achromatic mapping between the Magnifying Glass and Fresnel Corrector, this crucial process works simultaneously for all of the harmonic regions.

The introduction of multi-band lenses therefore enables the full Eyeglass telescope to (simultaneously) deliver diffraction-limited performance at multiple spectral windows, centered about each of the harmonic wavelengths. Each of these spectral windows has the same fractional bandwidth, set by the Eyepiece/Magnifying Glass aperture ratio. The fact that the window's relative widths stay constant, while their central wavelengths are (for increasing harmonics) getting closer together means that eventually the windows overlap; for all higher harmonics the windows merge together, resulting in fully-continuous spectral coverage for all wavelengths below some value. In this fashion, we have designed Eyeglass telescopes (for astronomical applications) which are diffraction-limited from $0.4-5.0 \mu \mathrm{ms}$.[1]

\section{FABRICATION and FIELDING}

Optically designing high-precision, broad band-width diffractive telescopes is just an initial step; they must still be implemented. We have to be able to fabricate large, 25-100 meter diffractive lenses, launch them into space, and then deploy and operate them there.

To be suitable, the lens material must be transparent, and must function during exposure to vacuum, meteoroids, sunlight, and radiation; it must not only physically survive, but must do so without significant darkening or dimensional changes. At present, the leading candidates for Eyeglass lenses are sheets of glass or silica, or films of polymers such as CP1 or other fluorinated polyimides. The advantage of the inorganics is their robustness and stability in space, that of the organics is that they are not brittle and are readily available in sub-mil thicknesses.

In forming the chosen material into a large diffractive lens, we can take advantage of two things; the required feature sizes are large, and the lens can (if necessary) be seamed together from smaller sections.

The smallest zone in a diffractive lens is given by $2 \lambda \mathrm{f} / \#$. Since the large diffractive lens is optically slow, $\mathrm{f} / \# \approx 100$, the smallest zones will be $\approx 100 \mu \mathrm{m}$, easily achievable with current technology. The challenge is presented by two other tolerances; the need to control the phase thickness of the lens material, and to place the Fresnel zones in their correct radial positions. In order to achieve $\lambda / 10$ imaging, the thickness profile (pattern plus substrate) of the film must be accurate (for typical materials) to $\lambda / 5$; for visible light this is about $0.1 \mu \mathrm{m}$. Note that this is purely a manufacturing challenge, fielding issues will primarily change the position of the film, not its thickness. So, in addition to applying a diffractive phase profile to the surface of the membrane, we must generally make it uniform thickness as well. Placing the Fresnel rings in their proper radial positions poses both fabrication and fielding challenges. The former is difficult but achievable, requiring both good metrology and thermal control during the lens fabrication; insuring that the rings remain in the proper absolute locations once the lens is in space will be more challenging.

The smoothing and patterning method utilized depends on the choice of lens material. While many options exist, the ones we are concentrating on at present are etching for glass and laser ablation for polymers. Glass and silica can be controllably etched by dilute solutions of hydrofluoric acid; smoothing can be done by closed-loop spatial control of the etching, while diffractive patterns can be emplaced by lithography. Polymers can be both smoothed and 
patterned by pulsed laser ablation, either with few-shake pulses from UV lasers, or much shorter pulses from visible or near- $\mathbb{R}$ lasers.

Packaging-for-launch is one of the fundamental challenges for any large aperture space telescope. In principle, since our diffractive lens is flat and thin, packaging ought to be simple. In practice, this is not the case, since neither glass nor polymer films can be folded tightly (glass breaks and polymers crease). Simple 1-D rug-roll methods work well up to $\sim 10$ meter sizes, but for 25-100 m apertures, fully 2-D packaging is required. For such sizes, we are considering a number of options, including in-situ assembly of flat panels, truss-mounted strips (packaged as 1$\mathrm{D}$ rolls), and origami- or umbrella-like folded lenses.

Once the lens reaches space, it must be deployed and held in shape. For Eyeglass, the desired shape is simply a flat disk, which is inherently simpler to form and maintain than are precisely curved reflecting mirrors, particularly given the loose (near-centimeter) flatness levels adequate for a slow Fresnel lens.

There is, however, one substantial challenge involved in fielding the Magnifying Glass lens; namely that the Fresnel zones must be in the correct radial locations. After being deployed in space, the Magnifying Glass lens will be kept flat by being held in tension. This flattens out elastic packaging wrinkles and raises the membrane's out-of-plane vibrational frequencies, making it less floppy. The dominant challenge in meeting the in-plane radial tolerances is not due to this stretching, (which is predictable and hence can be allowed for when writing the Fresnel pattern), nor is it from the presence of manufacturing seams; the largest distortions instead come from thermal strains. It is essential for the Magnifying Glass to be thermally "clean", that is, to avoid gradients in heating or in material properties that lead to nonuniform thermal stretching. Uniform temperature changes are not a problem, since they lead only to areally uniform stretching. For a Fresnel lens this just changes the focal length and hence is accommodated by a slight motion of the Eyepiece vehicle. Since astronomical Eyeglass telescopes will generally be placed behind a sunshield, it must be designed to produce as uniform as possible a post-shield irradiation profile. In addition, the lens material itself should have a low CTE to minimize the effect of nonuniform irradiation; this favors inorganics or advanced (TFDB-based) polyimides rather than glass or CP1-like polyimides. In addition to minimizing thermal distortions caused by the lens membrane, we should also avoid introducing such distortions from the lens-tensioning mechanism. Axial rotation is a particularly simple tensioning mechanism which is both selfdeploying and thermally "clean"; it requires only the optical membrane, with no extrinsic support structure. Other, truss-based, tensioning approaches can be used, but these must be designed to avoid thermally distorting the lens.

\section{FIVE METER LENS FABRICATION}

At present, we are engaged in building a large, 5 meter aperture, diffractive lens, it will have a 250 meter focal length for $600 \mathrm{~nm}$ light. This sized lens is a technological stepping-stone between the current (sub-meter) state-of-the-art, and the large $(25-100 \mathrm{~m})$ apertures desired for future space telescopes. While this lens is not intended to be fully space-rated, it is designed with this application in mind; the lens is made from a space suitable material (glass), will be lightweight $\left(1.6 \mathrm{~kg} / \mathrm{m}^{2}\right)$, and foldable.

Rather than building a full-sized, monolithic lens, we will instead create the overall lens by forming, and then joining together, a number of smaller lens segments. The virtue of this approach is that it splits an extremely difficult task into two, much more tractable, parts production of the lens panels and their precision assembly into a large lens. 
Before embarking on the segmented lens approach for our 5 meter lens, we demonstrated this approach at smaller (6 segment, $75 \mathrm{~cm}$ aperture) scale. The goal was to prove that diffractive subpanels could be independently patterned and then aligned and assembled together precisely enough to act as a coherent full-aperture lens; both before and after folding. The details of the fabrication, alignment, and optical testing are presented elsewhere[4]. In Figure 5, we show the 6segment lens in its mount, the lens while partially folded, and when fully folded. In Figure 6, we show the lens's diffraction-limited focal spot and PSF, both before and after the lens was folded.
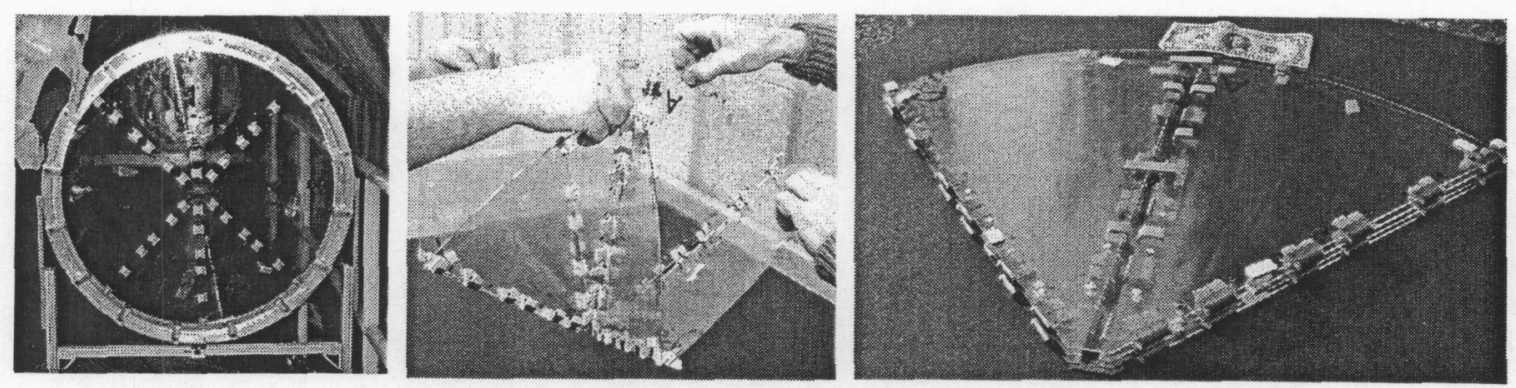

Figure 5: Six-segment diffractive lens: In mount, Being folded, Folded-up

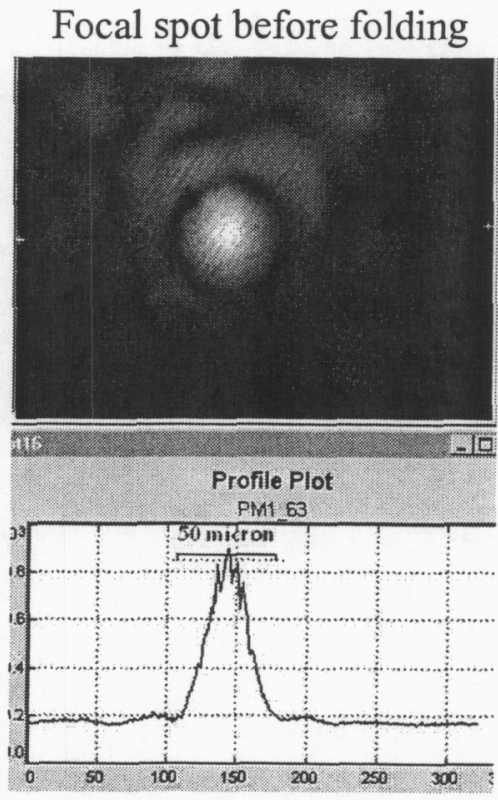

Focal spot after folding and unfolding

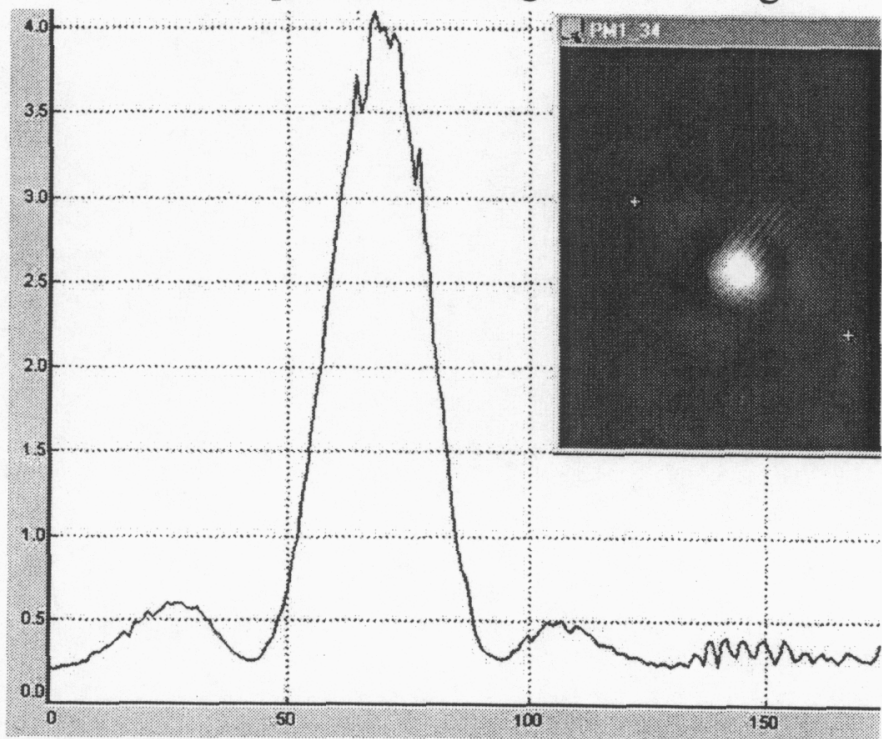

Figure 6: Focal spots of six-segment diffractive lens: Before and after folding

Having demonstrating the feasibility of segmented diffractive lenses, we are now engaged in scaling this approach up to a 5 meter aperture. The layout we have adopted is shown in Figure 7; it contains 72 lens panels in 8-fold symmetry. This foldable origami-layout was designed by Robert Lang; the folding/unfolding sequence for a similar 9-fold layout is shown in Figure 7. 

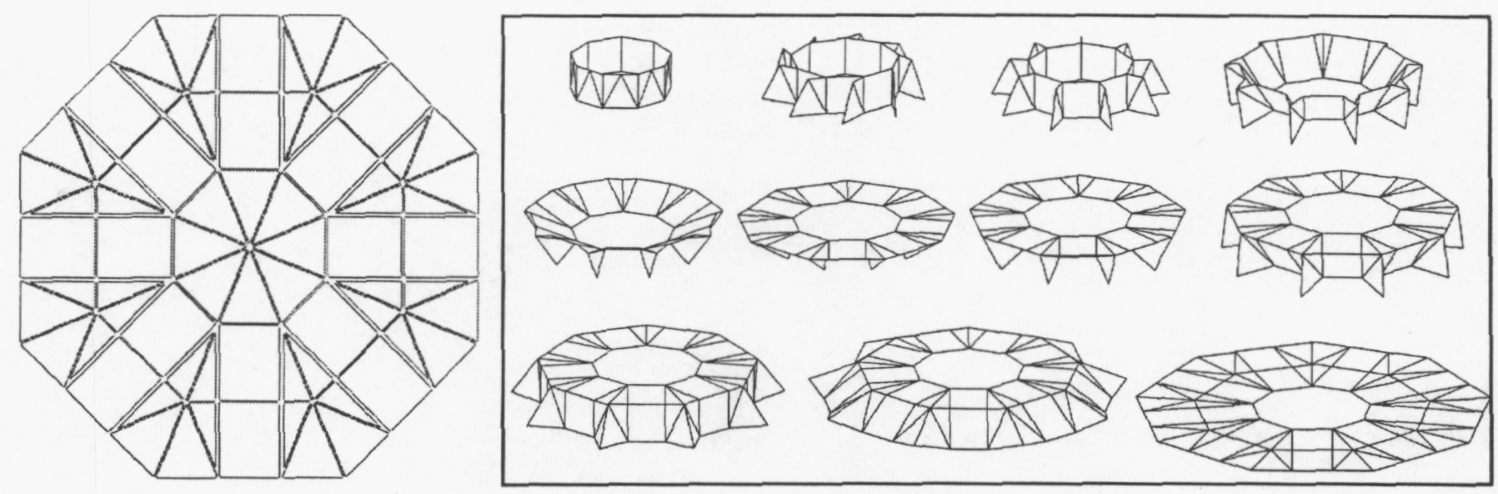

Figure 7: Layout and folding sequence for LLNL's 5 meter diffractive lens

We are building this lens out of thin sheets of glass, rather than silica or polymers. While polymers will likely prove lighter-weight and easier to launch, we chose glass for now, since we have more experience in smoothing and patterning it than we do with polymers. Silica is a better material than glass in many respects (transparency, radiation-resistance, CTE, etching simplicity), but we chose glass for now because we can affordably purchase meter-sized thin-sheets of glass (but not of silica). There are a number of commercially available varieties of thin-glass sheets; we are using $700 \mu \mathrm{m}$ thick sheets of Corning 1737 for our lens.

The as-purchased glass sheets must be optically processed into 72 separate lens panels. This involves two tasks, optical smoothing and diffractive patterning. It is most convenient to perform this processing on 40 identically-sized rectangular plates, rather than directly on the 72 final panels. The panel-to-plate groupings are shown in Figure 8. The diffractive profiles are emplaced on the glass by lithography, using masks and etching. This basic process is illustrated in Figure 9 for a single-mask, binary-profile pattern; Livermore's Diffractive Optics Group has the capability of making meter-sized diffractive optics, and has made 16-level, four-mask optics using such lithography. Multi-level diffractive profiles are important for lenses having high throughput and multi-band spectral performance, but our current, first generation, lens is being patterned with a simpler binary phase profile.

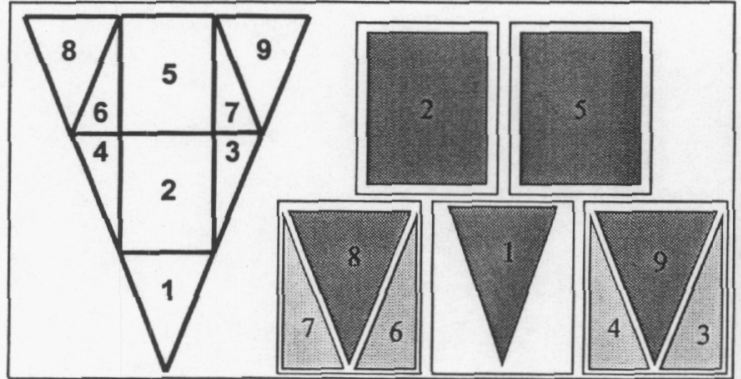

Figure 8: Panel-plate assignments

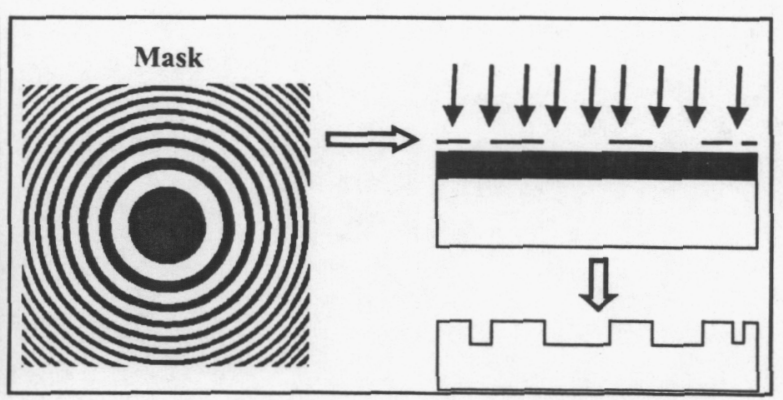

Figure 9: Lithographic patterning

The greatest drawback to using as-bought thin-glass sheets in a lens, is that they must be smoothed to a uniform (and constant) thickness. The typical wavefront of a Corning 1737 sheet is shown in Figure 10; it has quasi-1D thickness ripples with a depth of about 10 microns and spatial period of about $10 \mathrm{~cm}$. In order to be usable for a diffraction-limited lens these ripples must be removed. 


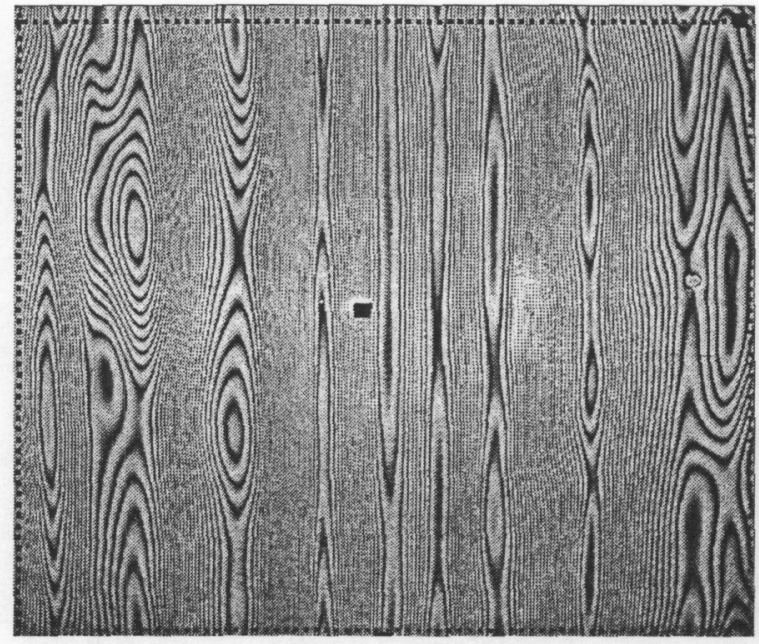

Figure 10: Wavefront errors in as-bought glass

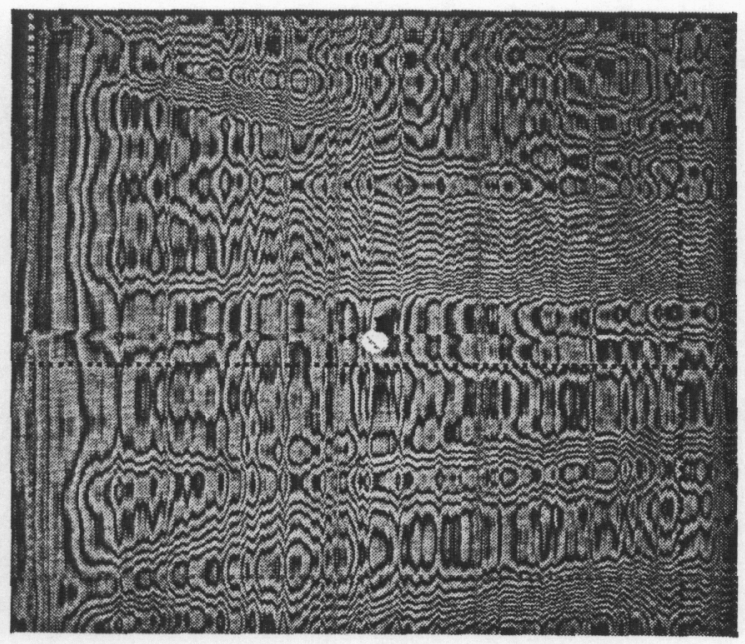

Figure 11: Wavefront after 1-D smoothing

Conventional finishing techniques like grind and polish are not suitable for flattening thin glass sheets. Moreover, non-destructive techniques such as ion milling are prohibitively expensive for large areas. In order to perform rapid, inexpensive smoothing of these thin (and delicate) glass sheets, LLNL has developed a new, spatially controlled etching method[5]. Etchant (containing dilute HF acid) is locally delivered to the glass though a small orifice in a tool-head, and (using the Marangoni effect) removed again; this creates a small, well-localized, pool of etchant. Using real-time feedback (from interferometric measurements of the glass thickness), the location of the tool-head (and hence the etchant) is moved across the surface of the glass, spending more dwell-time near peaks than valleys. After repeated measurements and transits, the glass is smoothed to the desired profile (either flat or non-flat). We have built and operated point-tool etchers which are capable of fully smoothing the 2-D ripples in thin-glass sheets. However, since the etchant is, at any given time, acting only on a small fraction of the sheet, this process is relatively slow. To speed-up the smoothing, we have built a line-etcher, exploiting the quasi-1D nature of the ripples evident in Figure 10. Results of this machine on an $80 \mathrm{~cm} \mathrm{x} 80 \mathrm{~cm}$ sheet of glass are shown in Figure 11 (view is rotated $90^{\circ}$ from that of Figure 10). Along a reference line, the glass thickness has been made uniform to better than $100 \mathrm{~nm}$.

However, because this etching tool is purely 1-D, the sheet does have 2-3 $\mu \mathrm{ms}$ of residual 2-D ripple. We have designed, and are currently fabricating, thermally-based 2-D etchers to rapidly remove such ripples.

Once the plates have been smoothed, patterned and cut into lens panels, they have to be precision assembled into a 5-m Fresnel lens. In order to achieve $\lambda / 10$ wavefront quality, we need $6 \mu \mathrm{m}$ radial positioning accuracy over the entire 5 meter aperture. While the absolute accuracy required for our 5 meter lens is the same as that of the $75 \mathrm{~cm}$ one, the relative accuracy is, of course, about 6.5 times greater. Achieving this large improvement in relative alignment accuracy, i.e., attaining $6 \mu \mathrm{m}$ precision over a 5 meter span instead of a $75 \mathrm{~cm}$ one, constitutes a major challenge to the assembly of our 5 meter lens.

As was done in the 75 -cm folding lens case, we will use a fiducial-based technique for aligning lens panels. During the generation of the mask used to apply the lens panel's diffractive surface profiles, we also write alignment fiducials around the borders of each panel. The panels are assembled one-at-a-time, aligned to their neighbors via these precisely-placed fiducials rather 
than the, much less precise, physical borders of the cut panels. Modeling of error accumulation during panel-by-panel assembly, shows that 1-2 $\mu \mathrm{m}$ fiducial alignment accuracies will be adequate to achieve our desired lens precision.
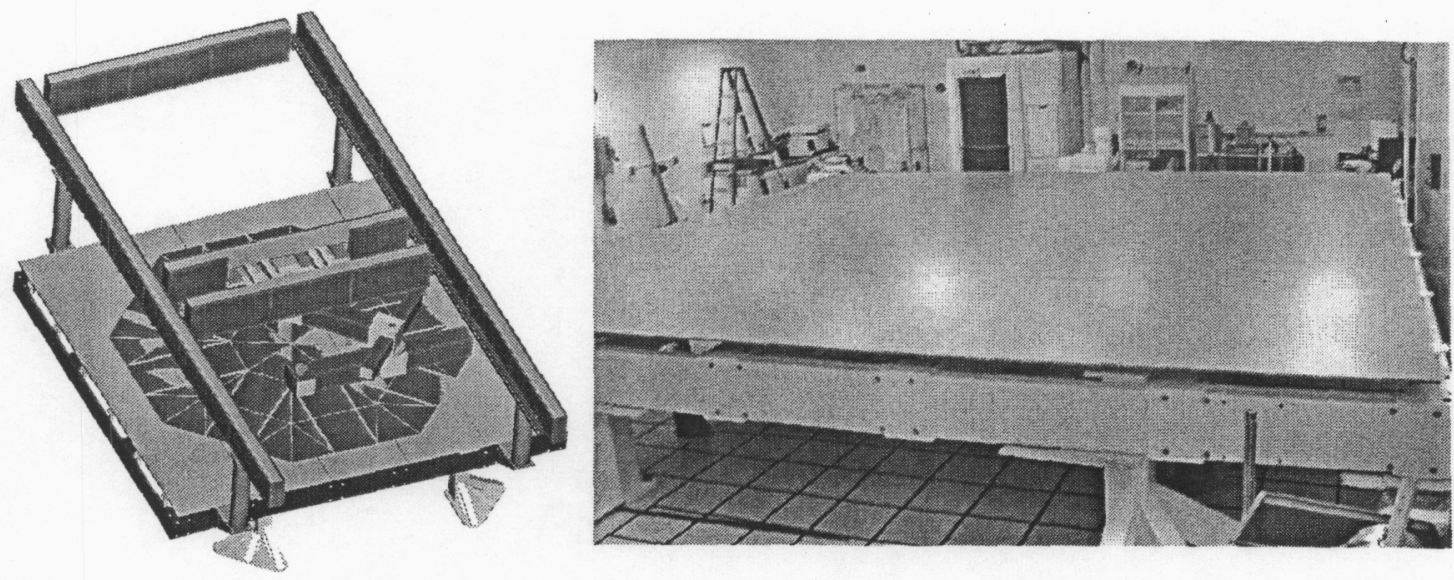

Figure 12: Lens assembly station: $\mathrm{CAD}$ view and partially fabricated

Lens assembly errors accumulate from a number of effects, including errors in placing and measuring fiducials, differential thermal expansion, imprecise panel placement, height differences across seams, etc. In order to deal with these error sources and to assemble a precise lens, we are building a semi-automated lens assembly station. Machine-based assembly will allow us to deal with the large size of the lens and the large number of lens panels, while (most critically) maintaining a thermally controlled environment. Figure 12 shows a $\mathrm{CAD}$ illustration of the assembly station, and a photograph of the currently assembled work surface of this machine.

The lens is assembled on the upper surface of a thermally controlled, vibrationally-isolated work-table. Thermal control is achieved by placing the assembly station within an insulated enclosure, and by incorporating an actively controlled heat exchanger just under the surface of the table. The glass panels are placed, aligned, and seamed on the table by use of a 2-D full-access delivery arm and a set of high-precision metrology and positioning tools. The 2-D arm serves to deliver the glass lens panels and the precision-assembly tools to their required locations on the table with an accuracy of $\sim 500 \mu \mathrm{m}$. The final $\mu \mathrm{m}$-level lens-assembly accuracy comes not from this 2-D delivery arm, but rather from the high-precision tools (a fiducial-looker and a positiontweaker) which it delivers. The assembly process will be as follows; first the 2-D arm delivers all of the lens panels to near (within the FOV of the fiducial-looker) their proper locations. At this point, the station's thermal control system is engaged, and the lens and work surface temperature is thereafter maintained with less than $0.1^{\circ} \mathrm{C}$ uniformity. Next, begins a panel-by-panel assembly process, in which one panel at a time is precision-aligned-with and attached-to its predecessors. Each of these steps begins with the 2-D arm delivering a panel-tweaker to one of the borders between the already-assembled lens and the new panel. After the tweaker has been delivered and grabs both parts, the arm delivers a fiducial-looker and carries it along the interface inspecting fiducials. This tool has 4 separate microscope-mounted cameras, held in precise registration. Each inspection involves simultaneously looking at two fiducials on the new panel and 2 on its already assembled neighbor; looking at 4 fiducials at once makes the inter-panel measurement insensitive to the position and orientation of the arm-mounted looker. After all fiducial groups have been inspected, a least-squares algorithm computes the proper shift and rotation of the new panel in order to bring it into alignment with the previously assembled portion of the lens. This command is sent to the panel-tweaker, which delivers the 3-DOF shift and rotation. After remeasurement 
confirms a correct alignment, a seamer-tool on the 2-D arm applies a series of thin-metal films (2 mil foils made from Elgiloy, a very high elastic-strain alloy) across the joint, connecting the newly aligned panel into place. The 2-D arm then moves the tweaker and looker to the next panel to be attached, and the assembly proceeds until complete.

We expect to complete the fabrication of the 5 meter diffractive lens this year. At present, $60 \%$ of the lens panels have been diffractively patterned. All of the hardware for the assembly station has been purchased, and it is in the process of being built and tested; the precision looker and tweaker are also near completion. While the 5 meter lens we are building is still much smaller than the 25-100 meter lenses which will be used for space telescopes, it should serve as an important milestone along the path towards such large aperture devices.

This research was performed under the auspices of the U.S. Department of Energy by Lawrence Livermore National Laboratory under contract W-7405-Eng-48. The authors acknowledge excellent contributions from Jerry Britten, Jim Early, Curly Hoaglan, Blake Myers, Jim Peterson, Paula Smith, Phil Steffan, Leslie Summers, Ian Thomas, and John Toeppen.

\section{REFERENCES}

[1] R. A. Hyde, 'Eyeglass. 1. Very large aperture diffractive telescopes,' Appl. Opt. 38 (19), 4198-4212 (1998).

[2] I. M. Barton, J. A. Britten, S. N. Dixit, L. J. Summers, I. M. Thomas, M. C. Rushford, K. Lu, R. A. Hyde and M. D. Perry, 'Fabrication of large-aperture, light weight diffractive lenses for use in space,' Appl. Opt. 40 447-451 (2001).

[3] R. A. Hyde, 'Eyeglass, A large-aperture space telescope,' UCRL-ID-136262, LLNL (2000).

[4] S. N. Dixit, R. A. Hyde, A. H. Weisberg, J. Early, M. C. Rushford, and J. A. Britten, 'Development of large-aperture, light weight Fresnel lenses for Gossamer space telescopes’ UCRL-JC-147407, LLNL (2002).

[5] M. C. Rushford, J. A. Britten, C. R. Hoaglan, I. M. Thomas, L. J. Summers and S. N. Dixit,'Wet-etch figuring: Optical surfacing by controlled application of etchant solution using the Marangoni effect,' Proceedings SPIE 4451, 249 - 258 (2001). 